\title{
Differential expression of miRNAs in esophageal cancer tissue
}

\author{
SHANG-GUO LIU ${ }^{1}$, XIU-GUANG QIN $^{1}$, BAO-SHENG ZHAO ${ }^{1}$, BO QI ${ }^{1}$, WEN-JIAN YAO ${ }^{1}$, \\ TIAN-YUN WANG ${ }^{2}$, HAN-CHEN LI ${ }^{1}$ and XIANG-NAN WU ${ }^{2}$ \\ ${ }^{1}$ Department of Thoracic Surgery, The First Affiliated Hospital of Xinxiang Medical University, Weihui 453100; \\ ${ }^{2}$ Department of Biochemistry and Molecular Biology, Xinxiang Medical University, Henan, Xinxiang 453003, P.R. China
}

Received December 7, 2012; Accepted February 26, 2013

DOI: $10.3892 / \mathrm{ol} .2013 .1251$

\begin{abstract}
The aim of this study was to explore the association of specific microRNAs (miRNAs) with the development of esophageal cancer (EC) and to identify new molecular markers for EC by analyzing the expression profiles of miRNAs in EC tissues. The expression profiles of miRNAs in paired EC and paracancerous normal tissues were detected and bioinformatically analyzed using miRNA assays. The outcomes were validated using real-time polymerase chain reaction. The miRNA assays revealed a total of 60 differentially expressed miRNAs in the EC tissues compared with those in the paracancerous normal tissues. Among them, 51 had doubled or more than doubled their expression levels and 9 had halved their expression levels. The most markedly upregulated miRNAs were hsa-miR-15a, hsa-miR-28-3p, hsa-miR-31, hsa-miR-99b, hsa-miR-101, hsa-miR-130a, hsa-miR-143, hsa-miR-196b, hsa-miR-200a, hsa-miR-210, hsa-miR-452 and hsa-miR-27a, whereas the most markedly downregulated miRNAs included hsa-miR-30b, hsa-miR-223, hsa-miR-454, hsa-miR-486, hsa-miR-574-3p and hsa-miR-126. Specific miRNA expression profiles exist in EC tissues and may serve as novel EC molecular markers.
\end{abstract}

\section{Introduction}

Esophageal cancer (EC) is one of the six most common malignancies worldwide, with a higher incidence in males than females (1). In China, EC has a mortality rate of 17.38/100,000 and ranks fourth among malignancies following gastric cancer, lung cancer and hepatocarcinoma. EC has an insidious onset; therefore, most patients have lapsed into advanced stage disease by the time of final diagnosis. The first-line treatment method for EC is currently a combination of surgery, chemo-

Correspondence to: Professor Bao-Sheng Zhao, Department of Thoracic Surgery, The First Affiliated Hospital of Xinxiang Medical University, No. 88 Jiankang Road, Weihui 453100, P.R. China

E-mail: zhaobscn@126.com

Key words: microRNA, expression profile, esophageal cancer, gene chip, real-time polymerase chain reaction therapy and radiotherapy. However, this method only achieves a five-year survival rate of $5-10 \%$. Within one year of the final diagnosis, $75 \%$ of patients succumb to EC (2). Therefore, identifying biological markers for EC to improve the early diagnosis rate and developing gene therapies is important for reducing the mortality rate of EC.

microRNAs (miRNAs) are a class of conservative single-stranded non-coding RNAs, composed of 17-25 ribonucleotides (3). Previous studies have shown that miRNA is involved in numerous physiological processes of cell regulation, including differentiation, proliferation, apoptosis and metabolism, and is also important in the development of cancer. A number of miRNAs function as oncogenes, while others may function as anti-oncogenes. Therefore, miRNAs have the potential to become new biological markers for EC and to be applied in the diagnosis, prognosis and targeted treatment of EC (4).

Tumors are subject to complicated medical conditions. The majority of tumors develop concurrently with the overexpression of oncogenes and/or the loss of anti-oncogenic expression. Almost half of human miRNAs are localized in tumor-associated genetic or fragile regions, including amplification, loss of heterozygosity, fragile and oncogene or anti-oncogene breakpoint regions (5). This suggests that miRNA has a role in the development of tumors. Studies have demonstrated that numerous miRNAs are directly involved in the initiation and development of EC and that their expression is correlated with the diagnosis, staging, progression and prognosis of EC (6-10).

Although studies have shown that miRNAs are important in the initiation, development, invasion and metastasis of tumors, their underlying mechanisms of action remain unknown. Whether the abnormal expression of miRNA is subject to the premise of tumorigenesis or its consequence, the mechanisms via which tissue-specific miRNAs act on different genes and the various factors which may regulate miRNA expression remain to be explored.

Binzhou and adjacent areas, including Huixian and Anyang in the Henan province of China, have a high incidence and mortality rate for EC. At present, EC remains the major cause of tumor-associated mortality in these areas (11). Since the molecular mechanisms underlying the initiation and development of EC remain unclear, a sensitive and specific molecular marker for the early diagnosis of EC has not yet been identified. This may be one of the major reasons for the 
Table I. miRNA expression in esophageal cancer and normal tissue.

\begin{tabular}{|c|c|c|c|c|}
\hline miRNA & $\begin{array}{l}\text { Esophageal cancer } \\
\text { tissue }\end{array}$ & Normal tissue & $\begin{array}{l}\text { Esophageal cancer tissue/ } \\
\text { normal tissue regulation }\end{array}$ & Fold \\
\hline hsa-miR-15a & 0.0000176246 & 0.0002582749 & Up & 22.61393059 \\
\hline hsa-miR-18a & 0.0000045344 & 0.0000220907 & Up & 5.974504433 \\
\hline hsa-miR-27b & 0.0001433345 & 0.0217867113 & Up & 6.007352154 \\
\hline hsa-miR-28-3p & 0.0000363064 & 0.0001763386 & $\mathrm{Up}$ & 24.12986015 \\
\hline hsa-miR-28 & 0.0000356773 & 0.0007026103 & Up & 12.20077435 \\
\hline hsa-miR-29b & 0.0000045110 & 0.0000446240 & Up & 6.058264764 \\
\hline hsa-miR-29c & 0.0000022294 & 0.0000109384 & Up & 10.6702369 \\
\hline hsa-miR-31 & 0.0000022218 & 0.0000152100 & Up & 20.82046385 \\
\hline hsa-miR-92a & 0.0001413414 & 0.0014162922 & Up & 14.68638018 \\
\hline mmu-miR-93 & 0.0000088895 & 0.0001243419 & $\mathrm{Up}$ & 6.011333249 \\
\hline hsa-miR-99a & 0.0000359286 & 0.0001740999 & $\mathrm{Up}$ & 7.386481816 \\
\hline hsa-miR-99b & 0.0000699830 & 0.0004923074 & $\mathrm{Up}$ & 24.24865292 \\
\hline hsa-miR-100 & 0.0000047146 & 0.0001007295 & $\mathrm{Up}$ & 6.025744617 \\
\hline hsa-miR-101 & 0.0000713869 & 0.0003512175 & Up & 21.61277146 \\
\hline hsa-miR-106b & 0.0000044494 & 0.0000598508 & $\mathrm{Up}$ & 11.79241078 \\
\hline hsa-miR-125b & 0.0000363742 & 0.0003481664 & $\mathrm{Up}$ & 10.49672038 \\
\hline hsa-miR-130a & 0.0000734506 & 0.0004906931 & $\mathrm{Up}$ & 29.05240133 \\
\hline hsa-miR-136 & 0.0000021992 & 0.0000609989 & $\mathrm{Up}$ & 10.89325593 \\
\hline mmu-miR-140 & 0.0000010873 & 0.0000076960 & $\mathrm{Up}$ & 5.975879236 \\
\hline hsa-miR-143 & 0.0000359259 & 0.0001759426 & Up & 23.88130556 \\
\hline mmu-miR-187 & 0.0002912525 & 0.0056576959 & $\mathrm{Up}$ & 9.763740353 \\
\hline hsa-miR-193a-3p & 0.0000393223 & 0.0002445030 & $\mathrm{Up}$ & 2.254945919 \\
\hline hsa-miR-193b & 0.0000023824 & 0.0000053720 & Up & 11.45048471 \\
\hline hsa-miR-196b & 0.0002979137 & 0.0028274519 & Up & 20.31581999 \\
\hline hsa-miR-200a & 0.0000022279 & 0.0000219849 & Up & 20.52730198 \\
\hline hsa-miR-210 & 0.0000371109 & 0.0004927418 & Up & 29.63419938 \\
\hline hsa-miR-218 & 0.0001432138 & 0.0040121562 & Up & 10.84716204 \\
\hline hsa-miR-339-3p & 0.0000179084 & 0.0001242423 & Up & 11.86043563 \\
\hline hsa-miR-155 & 0.0001468812 & 0.0014070302 & Up & 11.77170329 \\
\hline hsa-let-7b & 0.0001386657 & 0.0006844583 & Up & 6.118243605 \\
\hline hsa-miR-452 & 0.0000088236 & 0.0001757150 & Up & 24.59062971 \\
\hline hsa-miR-493 & 0.0000009831 & 0.0000109190 & Up & 11.11498543 \\
\hline hsa-miR-500 & 0.0000023069 & 0.0000154820 & Up & 10.41188492 \\
\hline hsa-miR-539 & 0.0000022609 & 0.0000153559 & Up & 10.39296695 \\
\hline hsa-miR-652 & 0.0000011157 & 0.0000306479 & Up & 19.51122157 \\
\hline hsa-miR-708 & 0.0000022561 & 0.0000213276 & Up & 10.27393174 \\
\hline hsa-miR-30a-5p & 0.0000187059 & 0.0001242389 & $\mathrm{Up}$ & 18.52588049 \\
\hline hsa-miR-622 & 0.0000034524 & 0.0000211653 & Up & 6.211150264 \\
\hline hsa-miR-572 & 0.0000031769 & 0.0000409741 & Up & 13.08208849 \\
\hline hsa-miR-770-5p & 0.0000127369 & 0.0001143333 & $\mathrm{Up}$ & 10.29941178 \\
\hline hsa-miR-99a & 0.0000032457 & 0.0000142938 & Up & 5.045521643 \\
\hline hsa-miR-145 & 0.0000016463 & 0.0000141523 & Up & 8.73420763 \\
\hline hsa-miR-27b & 0.0000064869 & 0.0000809041 & $\mathrm{Up}$ & 12.68090337 \\
\hline hsa-miR-378 & 0.0002220957 & 0.0006670456 & Up & 3.050953829 \\
\hline hsa-miR-151-3p & 0.0000510329 & 0.0006649765 & Up & 18.30361098 \\
\hline hsa-miR-214 & 0.0000033395 & 0.0000575783 & $\mathrm{Up}$ & 19.56162482 \\
\hline hsa-miR-425 & 0.0000062023 & 0.0000410135 & Up & 6.704588903 \\
\hline hsa-miR-10b & 0.0000068262 & 0.0000578115 & Up & 9.649507561 \\
\hline hsa-miR-181c & 0.0000259252 & 0.0001144539 & $\mathrm{Up}$ & 5.042527682 \\
\hline hsa-miR-27a & 0.0000031041 & 0.0000812814 & Up & 26.62109487 \\
\hline hsa-miR-1254 & 0.0000067522 & 0.0000561043 & Up & 9.44394700 \\
\hline
\end{tabular}


Table I. Continued.

\begin{tabular}{|c|c|c|c|c|}
\hline miRNA & $\begin{array}{l}\text { Esophageal cancer } \\
\text { tissue }\end{array}$ & Normal tissue & $\begin{array}{l}\text { Esophageal cancer tissue/ } \\
\text { normal tissue regulation }\end{array}$ & Fold \\
\hline hsa-miR-30b & 0.0000716277 & 0.0000076840 & Down & 0.11270600 \\
\hline hsa-miR-223 & 0.0092950671 & 0.0001796576 & Down & 0.02408100 \\
\hline hsa-miR-454 & 0.0002824790 & 0.0000220106 & Down & 0.16349700 \\
\hline hsa-miR-486 & 0.0001542431 & 0.0000156434 & Down & 0.15936700 \\
\hline hsa-miR-574-3p & 0.0005999424 & 0.0000857490 & Down & 0.17865900 \\
\hline hsa-miR-126 & 0.0000509905 & 0.0000070536 & Down & 0.15685000 \\
\hline hsa-miR-34a & 0.0000064237 & 0.0000024902 & Down & 0.39429700 \\
\hline hsa-miR-625 & 0.0000135014 & 0.0000050833 & Down & 0.38285000 \\
\hline hsa-miR-1290 & 0.0000132930 & 0.0000052090 & Down & 0.39754900 \\
\hline
\end{tabular}

miRNA, microRNA.

high mortality rate and poor prognosis of this condition (12). In the current study, miRNA spectra of three pairs of EC and paracancerous normal tissues were detected. The differentially expressed miRNAs were analyzed. The aim of this study was to provide a basis for further exploration of the mechanisms underlying the development of $\mathrm{EC}$, in addition to determining molecular markers associated with EC for its early diagnosis and treatment.

\section{Materials and methods}

Clinical data. Three EC specimens from the First Affiliated Hospital of Xinxiang Medical University were collected after first resection of the primary lesions. Fresh normal mucous membranes of the esophagus at the same pathological stage, $8 \mathrm{~cm}$ from the border of the tumor tissues were collected for the control samples. All resected tissue samples were stored at $-80^{\circ} \mathrm{C}$ after freezing with liquid nitrogen. This study was conducted in accordance with the Declaration of Helsinki and with approval from the Ethics Committee of Xinxiang Medical University. Written informed consent was obtained from all participants.

Sample treatment and RNA extraction. Total RNA was extracted from the oncocytes in the sections using the TRIzol one-step method according to the manufacturer's instructions (Invitrogen, Carlsbad, CA, USA). Total RNA was collected according to the manufacturer's instructions in the Recover All Total Nucleic Acid Isolation kit TRIzol reagent (Applied Biosystems, Carlsbad, CA, USA). Light absorption values were read at 230, 260 and $280 \mathrm{~nm}$ using spectrophotometry to determine purity and density. Formaldehyde-agarose gel electrophoresis was performed for the $28 \mathrm{~S}: 18 \mathrm{~S}$ ratio to determine the purity and integrity of the total RNA sample.

Real-time quantitative reverse transcription polymerase chain reaction ( $R T-q P C R)$. miRNAs were quantitated using RT-qPCR. The Megaplex Pool reverse transcription system (Applied Biosystems) was prepared with a total volume of $4.5 \mu 1$, containing Megaplex ${ }^{\mathrm{TM}} \mathrm{RT}$ primers $(10 \mathrm{X})$ at $0.8 \mu 1$, dNTPs with dTTP $(100 \mathrm{mM})$ at $0.2 \mu \mathrm{l}$, MultiScribe ${ }^{\mathrm{TM}}$ reverse transcriptase (50 U/ $\mu \mathrm{l}$; Applied Biosystems) at $1.5 \mu 1$, 10X RT buffer at $0.8 \mu \mathrm{l}, \mathrm{MgCl}_{2}(25 \mathrm{mM})$ at $0.9 \mu \mathrm{l}$, RNase inhibitor $(20 \mathrm{U} / \mu \mathrm{l})$ at $0.1 \mu \mathrm{l}$ and nuclease-free water at $0.2 \mu \mathrm{l}$. The solution was reverse transcribed six times and then centrifuged. Total RNA at $3 \mu 1$ (1-350 ng) was added into the reaction tube, reverse transcribed six times, centrifuged and placed on ice for $5 \mathrm{~min}$. The PCR conditions consisted of 40 cycles of $16^{\circ} \mathrm{C}$ for $2 \mathrm{~min}, 42^{\circ} \mathrm{C}$ for $1 \mathrm{~min}$ and $50^{\circ} \mathrm{C}$ for $1 \mathrm{sec}$, followed by $85^{\circ} \mathrm{C}$ for 5 min and a termination step at $4^{\circ} \mathrm{C}$. The reverse transcription products were placed on ice.

The pre-amplification PCR system was prepared with a total volume of $25 \mu \mathrm{l}$, containing TaqMan ${ }^{\circledR}$ PreAmp Master Mix (2X; Applied Biosystems) at $12.5 \mu 1$, Megaplex ${ }^{\mathrm{TM}}$ PreAmp primers $(10 \mathrm{X})$ at $2.5 \mu \mathrm{l}$, RT product at $2.5 \mu \mathrm{l}$ and nuclease-free water at $7.5 \mu 1$. The solution was reverse transcribed six times, centrifuged and then placed on ice for $5 \mathrm{~min}$. The PCR conditions consisted of $95^{\circ} \mathrm{C}$ for $10 \mathrm{~min}, 12$ cycles of $55^{\circ} \mathrm{C}$ for $2 \mathrm{~min}$, $72^{\circ} \mathrm{C}$ for $2 \mathrm{~min}, 95^{\circ} \mathrm{C}$ for $15 \mathrm{sec}$ and $60^{\circ} \mathrm{C}$ for $4 \mathrm{~min}$, followed by $99.9^{\circ} \mathrm{C}$ for $10 \mathrm{~min}$ and a termination step at $4^{\circ} \mathrm{C}$.

TE $(0.1 \mathrm{X} ; \mathrm{pH} 8.0)$ at $75 \mu \mathrm{l}$ was added into the pre-amplification tube, reverse transcribed six times and then stored at $-20^{\circ} \mathrm{C}$. TaqMan universal PCR Master mix was swirled to blend.

Regents with a total volume of $900 \mu 1$, including TaqMan ${ }^{\circledR}$ universal PCR Master mix, No AmpErase ${ }^{\circledR}$ UNG (2X) at $450 \mu \mathrm{l}$, diluted PreAmp product at $9 \mu \mathrm{l}$ and nuclease-free water at $441 \mu \mathrm{l}$, were added into a $1.5-\mathrm{ml}$ centrifuge tube, reverse transcribed six times and centrifuged.

The PCR mix at $100 \mu \mathrm{l}$ was applied to each sampling point and then twice centrifuged at 1,200 rpm ( $1 \mathrm{~min}$ each time). The reaction conditions consisted of $94.5^{\circ} \mathrm{C}$ for $10 \mathrm{~min}, 40$ cycles of $97^{\circ} \mathrm{C}$ for $30 \mathrm{sec}$ and $59.7^{\circ} \mathrm{C}$ for $1 \mathrm{~min}$.

Statistical analysis. The U6 snRNA housekeeping gene was used as an internal reference. The relative quantitative method was used. Gene expression was quantitated based on the following formula: $\mathrm{F}=2^{-\Delta \Delta \mathrm{ct}}$ where $\Delta \Delta \mathrm{ct}=$ (ct mean of the target gene in the test sample - that of the housekeeping gene in the test sample) - (ct mean of the target gene in the control sample - that of the housekeeping gene in the control sample). A higher F-value indicates higher expression levels. 


\section{Results}

As shown in Table I, compared with the normal tissues, tumor tissues exhibited 60 differentially expressed miRNAs among the total 770. From the differentially expressed miRNAs, 51 were upregulated and 9 were downregulated. A number of miRNAs were upregulated $>20$ times, including hsa-miR15a, hsa-miR-28-3p, hsa-miR-31, hsa-miR-99b, hsa-miR-101, hsa-miR-130a, hsa-miR-143, hsa-miR-196b, hsa-miR-200a, hsa-miR-210, hsa-miR-452 and hsa-miR-27a, whereas those which were downregulated $>5$ times included hsa-miR-30b, hsa-miR-223, hsa-miR-454, hsa-miR-486, hsa-miR-574-3p and hsa-miR-126 (Table I).

\section{Discussion}

EC is a common malignancy and China is the country in which the highest morbidity and mortality rates for EC occur. The development of EC involves multiple factors, numerous genes and several stages. As most EC cases have progressed to advanced stage disease by the time of final diagnosis, EC patients always have a poor prognosis, with a five-year survival rate between 5 and 20\%. Previous studies have revealed that the majority of ECs develop concurrently with the overexpression of oncogenes and/or the loss of anti-oncogenic expression $(13,14)$. Almost half of miRNAs are localized at the fragile sites or in associated genetic regions of these genes, where they exert post-transcriptional control over the genes. Additionally, studies have shown that miRNAs tend to be expressed abnormally in tumor tissues $(15,16)$. miRNAs function as tumor-inhibiting or cancerogenic factors in the development of tumors and also have extensive application value for diagnosing and predicting the prognosis of tumors.

In this study, miRNA expression in the EC and normal tissues was determined using miRNA chip technology. EC-associated miRNA spectra were obtained. Significantly upregulated miRNAs in the EC tissues included hsa-miR-15a, hsa-miR-28-3p, hsa-miR-31, hsa-miR-99b, hsa-miR-101, hsa-miR-130a, hsa-miR-143, hsa-miR-196b, hsa-miR-200a, hsa-miR-210, hsa-miR-452 and hsa-miR-27a. miRNAs which were downregulated $>5$ times included hsa-miR-30b, hsa-miR-223, hsa-miR-454, hsa-miR-486, hsa-miR-574-3p and hsa-miR-126. The 31 miRNA spectra obtained from the EC tissues were further analyzed using microassay technology. Results showed that 60 miRNAs exhibited abnormal expression. Guo et al (9) have shown that three miRNAs (hsa-miR-25, hsa-miR-424 and hsa-miR-151) are upregulated and four miRNAs (hsa-miR-100, hsa-miR-99a, hsa-miR-29c and mmu-miR-140) are reduced in cancer tissue compared with normal tissue. The results of this study showed that hsa-miR-25 was upregulated one and a half times in the tumor tissues, which is consistent with the results of Guo et al. However, this study also showed that hsa-miR-100, hsa-miR-99a and hsa-miR-29c were markedly upregulated in the tumor tissues, which contradicts the results of Guo et al. This disagreement is possibly ascribed to the small sample size, source of materials and EC stage in this study. Zhang et al (17) concluded, based on the comparison between 209 EC and 140 control samples, that seven serum miRNAs (miR-10a, miR-22, miR-100, miR148b, miR-223, miR-133a and miR-127-3p) were significantly upregulated in the sera of esophageal squamous cell carcinoma patients compared with control individuals. This study also showed that the EC tissues exhibited upregulated miR-100 and miR-133a compared with normal tissues.

In conclusion, a total of 60 miRNAs were abnormally expressed in EC tissues compared with those in normal tissues. 51 miRNA fragments were upregulated $\geq 2$ times and 9 were downregulated to less than half. However, considering the small number of samples employed in this study, our results require confirmation using a larger sample size.

\section{References}

1. Enzinger PC and Mayer RJ: Esophageal cancer. N Engl J Med 349: 2241-2252, 2003.

2. Xing D, Tan W and Lin D: Genetic polymorphisms and susceptibility to esophageal cancer among Chinese population (review). Oncol Rep 10: 1615-1623, 2003.

3. Cho WC: OncomiRs: the discovery and progress of microRNAs in cancers. Mol Cancer 6: 60, 2007.

4. Matsushima K, Isomoto H, Kohno S and Nakao K: MicroRNAs and esophageal squamous cell carcinoma. Digestion 82: 138-144, 2010.

5. Calin GA, Sevignani C, Dumitru CD, et al: Human microRNA genes are frequently located at fragile sites and genomic regions involved in cancers. Proc Natl Acad Sci USA 101: 2999-3004, 2004.

6. Yang M, Liu R, Sheng J, et al: Differential expression profiles of microRNAs as potential biomarkers for the early diagnosis of esophageal squamous cell carcinoma. Oncol Rep 29: 169-176, 2013.

7. Lin RJ, Xiao DW, Liao LD, et al: MiR-142-3p as a potential prognostic biomarker for esophageal squamous cell carcinoma. J Surg Oncol 105: 175-182, 2012.

8. Xu X, Chen Z, Zhao X, et al: MicroRNA-25 promotes cell migration and invasion in esophageal squamous cell carcinoma. Biochem Biophys Res Commun 421: 640-645, 2012.

9. Guo Y, Chen Z, Zhang L, et al: Distinctive microRNA profiles relating to patient survival in esophageal squamous cell carcinoma. Cancer Res 68: 26-33, 2008.

10. Mathé EA, Nguyen GH, Bowman ED, et al: MicroRNA expression in squamous cell carcinoma and adenocarcinoma of the esophagus: associations with survival. Clin Cancer Res 15: 6192-6200, 2009.

11. Song PI, Liang H, Fan JH, Wei WQ, Wang GQ and Qiao YL: Long-term survival after esophagectomy for early esophageal squamous cell carcinoma in Linxian, China. J Surg Oncol 104: 176-180, 2011.

12. Sun X, Chen W, Chen Z, Wen D, Zhao D and He Y: Population-based case-control study on risk factors for esophageal cancer in five high-risk areas in China. Asian Pac J Cancer Prev 11: 1631-1636, 2010.

13. Hu YC, Lam KY, Law S, Wong J and Srivastava G. Identification of differentially expressed genes in esophageal squamous cell carcinoma (ESCC) by cDNA expression array: overexpression of Fra-1, Neogenin, Id-1, and CDC25B genes in ESCC. Clin Cancer Res 7: 2213-2221, 2001.

14. Li Y, Chen L, Nie CJ, Zeng TT, Liu H, Mao X, Qin Y, Zhu YH, $\mathrm{Fu}$ L and Guan XY: Downregulation of RBMS3 is associated with poor prognosis in esophageal squamous cell carcinoma. Cancer Res 19: 6106-6115, 2011.

15. Rosenfeld N, Aharonov R, Meiri E, et al: MicroRNAs accurately identify cancer tissue origin. Nat Biotechnol 26: 462-469, 2008.

16. Feber A, Xi L, Luketich JD, Pennathur A, et al: MicroRNA expression profiles of esophageal cancer. J Thorac Cardiovasc Surg 135: 255-260, 2008.

17. Zhang C, Wang C, Chen X, et al: Expression profile of microRNAs in serum: a fingerprint for esophageal squamous cell carcinoma. Clin Chem 56: 1871-1879, 2010. 